\title{
Novel strategy for treating neural disease
}

\author{
CHENG Lin \\ State Key Laboratory of Cell Biology, Institute of Biochemistry and Cell Biology, Shanghai Institutes for Biological Sciences, Chinese \\ Academy of Sciences, Shanghai 200031, China
}

Received May 12, 2014; accepted May 29, 2014; published online June 13, 2014

Citation: Cheng L. Novel strategy for treating neural disease. Sci China Life Sci, 2014, 57: 947-948, doi: 10.1007/s11427-014-4686-2

Traditional therapies for diseases of brain and spinal cord just alleviate poorly symptoms without effective therapy. Neural cell replacement may be a promising therapy for neurological diseases such as Alzheimer's disease, Parkinson's disease and amyotrophic lateral sclerosis. Among neural cells, neural progenitor cells (NPCs) with the ability of self-renewal and the potential of multi-lineage neural differentiation have been the most prospective cell candidate for replacement [1]. So far conventional protocols for generating NPCs, including differentiation of embryonic stem cells (ESCs) or of induced pluripotent stem cells (iP$\mathrm{SCs}$ ) into NPCs or direct derivation from brain tissues, are limited due to ethical and practical issues and safety concern. Recent achievement in converting somatic cells into neural cells for transplantation thus provides a new strategy for a better alternative. Induced neural cells (iNCs) include induced neural progenitor cells or induced neurons from fibroblasts, hepatocyte, astrocytes, or pericytes by introduction of exogenous factors; however, safety concern still remains. Therefore, generation of iNCs from somatic cells using only chemical or physical methods will offer a desirable and potentially safer patient-specific neural cells for treating neural disease.

Recently, a combination of small molecules was shown to be able to convert terminally differentiated cells into neural progenitor cells under physiological hypoxia in vitro [2]. Under hypoxic conditions, simultaneous inhibition of HDACs, GSK-3 $\beta$, and TGF- $\beta$ signaling pathway by respective inhibitors significantly upregulated expression level of

*Corresponding author (email: lcheng@ sibcb.ac.cn)
Sox2, which plays a key role in NPC induction [3]. Meantime, compact cell colonies positive for alkaline phosphatase activity were formed during the initial treatment. Homogenous chemical-induced neural progenitor cells (ciNPCs) were generated when these intermediate cells were cultured in neural expansion medium (lineage-specific culture conditions) followed by enrichment through several rounds of neurosphere culture. These ciNPCs converted from mouse embryonic fibroblasts resembled mouse brain-derived NPC properties regarding their proliferative and self-renewing abilities, gene expression profiles, and multipotency for different neuroectodermal lineages in vitro and in vivo. Most importantly, this chemical induction strategy is applicable for converting human urinary cells into neural progenitor cells, which not only demonstrates generality of the method but also holds good promise for clinical applications.

Compared with genetic approaches, direct lineagespecific conversion of differentiated somatic cells into NPCs achieved by chemical cocktails without introducing exogenous genes and passing through a pluripotent state [4] is definitely a more attractive strategy. It is much easier of administration and more practical for in vitro and in vivo applications. Unlike ESC or iPSC-based strategies in generating desired cell types, direct conversion approach is much faster and more efficient and even safer for cell therapy as it may avoid the risk of tumorigenesis inherent with pluripotent stem cells. Despite these apparent merits, a number of interesting questions still remain. For example, why these three signaling pathways are essentially linked to neural conversion and what the mechanisms underlying this repro- 


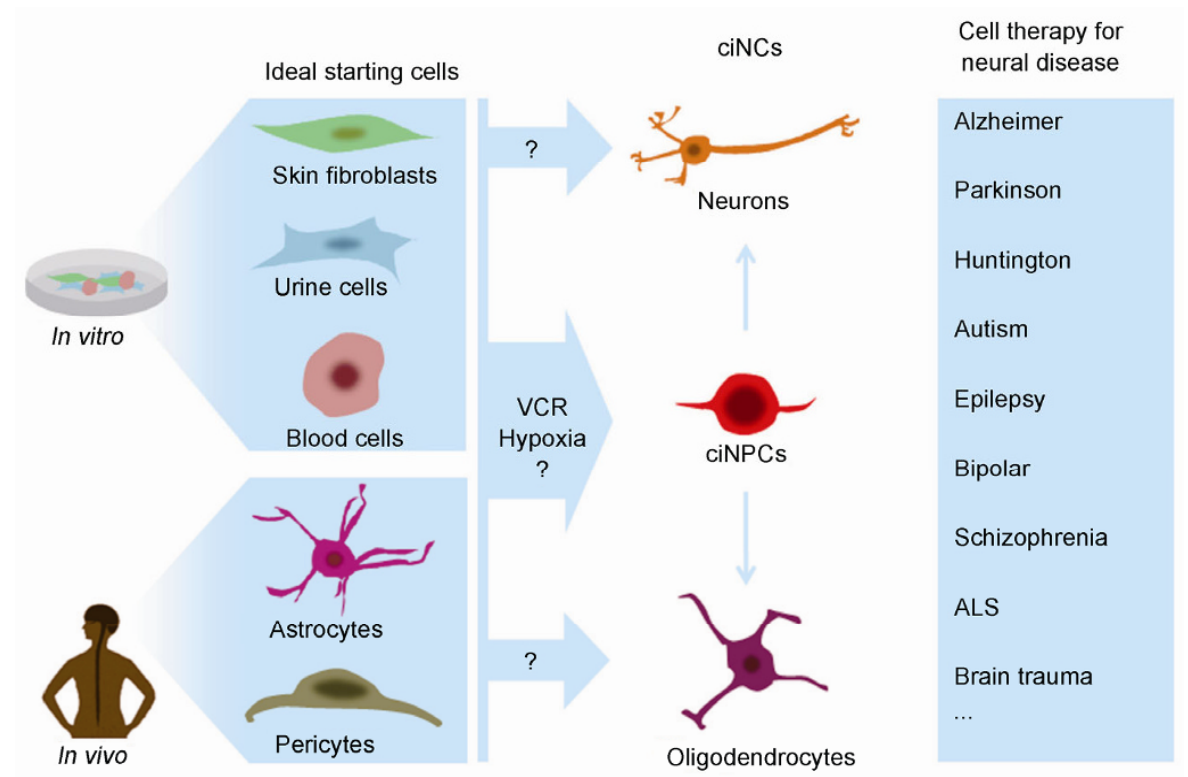

Figure 1 Direct conversion of various human cell types into induced neural cells by complete small molecules in vitro and in vivo. The ideal starting human cells for in vitro neural conversion would be skin fibroblasts, urine cells and blood cells, while the in vivo one might be astrocytes and pericytes. By complete chemical treatment without introducing exogenous genes, the desired chemical-induced neural cells (ciNCs) for cell therapy would be ciNPCs, neurons or oligodendrocytes to each type of neural diseases. Nevertheless, the optimal recipes of chemical compounds for each direct conversion still remain to be further investigated.

gramming are and how to erase the epigenetic memory of starting non-neural lineage cells. Considering the more accessible and abundant somatic cell types from human patients of different ages still include skin fibroblasts, blood cells and adipocytes, the feasibility and efficiency of generating ciNPCs in vitro from these cells still need to be investigated and optimized.

Based on current studies, it is of great interest to test whether the chemical cocktails would facilitate neural conversion in vivo and eventually lead to neuronal regeneration under physiological hypoxia. At this case, the ideal starting cells might be astrocytes or pericytes accumulated after nervous system injury or neurodegeneration, and the desired end cell types could be neural progenitor cells, subtype specific neurons, oligodendrocytes or Schwann cells, generally named as chemical-induced neural cells (ciNCs) (Figure 1). Since aging is accompanied by stem cell degeneration, administration of drug cocktails or even the herbal extracts (such as the traditional Chinese medicine) might help achieve neural conversion in vivo and even rejuvenation.

Except for the novel chemical methods of generating neural cells, physical strategy of promoting cell reprogramming has also been reported, such as three-dimensional culturing condition, growing substrates with microgrooves or treatment of stress. Even just acid-bath treatment of cells for a short time is reported recently to be able to reprogram somatic cells into pluripotent cells (still controversial) [5]. According to all these studies and similar theories from genetic induction, we hypothesize that treating cells with only these physical conditions might be able to reprogram cells into "plastic" state and further culturing the intermediate cells in lineage specific medium would generate desired target cells, including neural cells and even cells functioned in other germ layers, such as hematopoietic stem cells and cardiac stem cells.

1 Martino G, Pluchino S. The therapeutic potential of neural stem cells. Nat Rev Neurosci, 2006, 7: 395-406

2 Cheng L, Hu W, Qiu B, Zhao J, Yu Y, Guan W, Wang M, Yang W, Pei G. Generation of neural progenitor cells by chemical cocktails and hypoxia. Cell Res, 2014, doi: 10.1038/cr.2014.32

3 Ring KL, Tong LM, Balestra ME, Javier R, Andrews-Zwilling Y, Li G, Walker D, Zhang WR, Kreitzer AC, Huang YD. Direct reprogramming of mouse and human fibroblasts into multipotent neural stem cells with a single factor. Cell Stem Cell, 2012, 11: 100-109

4 Hou P, Li Y, Zhang X, Liu C, Guan J, Li H, Zhao T, Ye J, Yang W, Liu K. Pluripotent stem cells induced from mouse somatic cells by small-molecule compounds. Science, 2013, 341: 651-654

5 Obokata H, Wakayama T, Sasai Y, Kojima K, Vacnti MP, Niwa H, Yamato M, Vacnti CA. Stimulus-triggered fate conversion of somatic cells into pluripotency. Nature, 2014, 505: 641-647

Open Access This article is distributed under the terms of the Creative Commons Attribution License which permits any use, distribution, and reproduction in any medium, provided the original author(s) and source are credited. 